Cite this: J. Mater. Chem. A, 2013, 1, 14097

Received 29th August 2013 Accepted 3rd October 2013

DOI: 10.1039/c3ta13435k

www.rsc.org/MaterialsA

\section{One-pot synthesis of nitrogen-sulfur-co-doped carbons with tunable composition using a simple isothiocyanate ionic liquid $\dagger$}

\author{
Nina Fechler, ${ }^{*}$ Tim-Patrick Fellinger and Markus Antonietti \\ The commercially available ionic liquid (IL) 1-ethyl-3-methyl imidazolium thiocyanate (Emim-scn) is used as \\ a highly efficient precursor for the synthesis of nitrogen-sulfur-co-doped carbons. Mixtures with the ILS \\ 1-ethyl-3-methyl-imidazolium dicyanamide (Emim-dca) and 1-butyl-3-methyl-pyridinium dicyanamide \\ (Bmp-dca) enable tuning of the sulfur-content while the nitrogen incorporation is not hindered as such. \\ The whole procedure is compatible with the salt templating process, enabling the synthesis of \\ heterofunctional carbons with ultrahigh surface areas.
}

\section{Introduction}

One of the recent breakthroughs in materials chemistry is the manipulation of carbon-based compounds through the structural introduction of heteroatoms. Here, nitrogen is an established doping-atom and it is well known that its introduction into a pool of $\mathrm{sp}^{2}$-bonded graphitic carbon atoms leads to distinctly different carbon material properties. Improved electric conductivity and increased chemical stability are just two of the potential benefits. ${ }^{1}$ While surface-attached nitrogen in the form of functional groups mostly changes chemical properties and is therefore called chemical nitrogen, structural nitrogen (incorporated into the graphitic sheets) also shows high impact on the physical properties of the final carbon material. A very prominent property amplification achieved by such a nitrogen introduction is the increase of electric conductivity, ${ }^{2-5}$ which is caused by modification of the band structure of the material. ${ }^{6}$ The valence band is lowered, thus the material is chemically more stable, and the electron density at the Fermi level is increased. This has experimentally been shown to induce a metallic character in nitrogen-doped carbon nanotubes (CNTs). ${ }^{7}$ Practical observations could also be supported by quantum mechanical calculations by Carvalho et al. ${ }^{8}$ Therefore, nitrogen-doped carbons are sometimes even referred to as "noble-carbons" and have been successfully applied in a variety of applications, e.g. oxygen reduction reaction, $\mathrm{H}_{2} \mathrm{O}_{2}$-production or $\mathrm{CO}_{2}$ - and energy-storage. ${ }^{9-13}$ Besides nitrogen, there is

Max-Planck-Institute of Colloids and Interfaces, Department of Colloid Chemistry, Am Mühlenberg 1, 14476 Potsdam-Golm, Germany.E-mail: nina.fechler@mpikg.mpg.de $\uparrow$ Electronic supplementary information (ESI) available: Chemical structures of Emim-scn, Emim-dca and Bmp-dca; TGA-DSC of Emim-scn; XRD patterns of N-S-dC_IL_x_800; $\quad$ EDX $\quad$ of $\quad \mathrm{N}-\mathrm{S}-\mathrm{dC} \_I L \_2 \_800 ; \quad$ elemental analysis of N-S-dC_IL_2_SZ_3_800; TEMs and SEMs of N-S-dC_Bmp-dca_x_800. SEM of N-S-dC_IL_2_SZ_3_800. See DOI: 10.1039/c3ta13435k also evidence for an additional amplification of carbon materials through structural boron, phosphorus or sulfur atoms. ${ }^{\mathbf{1 4 - 1 9}}$ In particular, sulfur arouses interest, because first results suggest a synergistic interaction of the $\mathrm{N}$ - and S-dopants presumably due to an easier polarizability. This is supported by DFT calculations conducted by Qiao et al. which reveal the synergistic interaction of $\mathrm{N}$ - and S-co-doping to result from the redistribution of spin and charge densities in the HOMO structure. ${ }^{20}$ However, until now, the synthesis of heteroatom-codoped carbons remains challenging and new strategies are highly welcomed.

Recently, some groups employed carbonizable (e.g. cyano containing) ILs as a direct precursor for the synthesis of nitrogen, ${ }^{21-24}$ phosphorus ${ }^{25}$ or sulphur-doped carbons. ${ }^{24,26}$ ILs indeed have many practical advantages as a carbon precursor. ILs are almost non-volatile due to their salt-like character, and there is no need to apply a confining high pressure, rendering the synthesis highly facile and without larger technical efforts. $^{27,28}$ Carbons with nitrogen contents as high as $10.4 \%$ with an electronic conductivity superior to graphite and an oxidation stability higher than carbon nanotubes were obtained. This approach has recently been analyzed via careful mechanic analysis on the formation mechanism. ${ }^{21}$ In addition, ILs show strong interactions with inorganic surfaces and thereby very good wettability. That way, the precursors may be dip-coated, spun, printed, sprayed or templated towards nanostructures before or throughout conversion to the final nitrogen-doped carbon in simple inert gas ovens. ${ }^{22}$ The miscibility of ILs with ordinary salt was recently used to develop the technique of "salt templating", ${ }^{29}$ and materials with unusually high specific surface areas of up to $2000 \mathrm{~m}^{2} \mathrm{~g}^{-1}$ could be obtained. Depending on the type of salt, pore sizes in the range of engulfing ion pairs to nanometer sized ion clusters were obtained. 
ILs are chosen in a way that the cation already contains structural nitrogen, e.g. pyridinium, pyrrolidinium or imidazolium, while the anion preferentially carries cyano functionalities, because only few, and in particular cyano-containing ILs, give high carbon yields in the absence of nanoconfinement. ${ }^{24}$ The role of cyano-functions is therefore obviously a highly important one, permitting the first and essential step of formation of a resin which is preorganized and thermostable. ${ }^{14,30}$ The synthesis of nitrogen-sulfur-co-doped carbons with a tunable composition consequently relies on sulfur containing cations as thiazolium compounds, which are however still less available and convenient to use.

In this paper, we will illustrate the practical use of the isothiocyanate counterion, which is not only one of the most convenient chemical sources of covalent sulfur, but - due to its high polarizability - also enables structural fixation at comparably low temperatures by cycloaddition reactions with different $\mathrm{sp}^{2}$-compounds. Thus, added as a co-monomer, it does not compete with the simultaneous incorporation of structural nitrogen but enters the entire product formation process, resulting in nitrogen-sulfur-co-doped carbon materials. The sulfur content can be tuned by the amount of thiocyanate used in the initial precursor mixture, and high surface areas can be introduced by applying the salt templating approach.

\section{Experimental}

\section{Materials and methods}

Emim-scn (purity of $>95 \%$ ) and Emim-dca (purity of $>98 \%$ ) were purchased from IoLiTec and Bmp-dca from Merck (purity of $>98 \%$ ). Sodium chloride and zinc chloride (99\%) were acquired from Sigma Aldrich. All chemicals were used without further purification.

The eutectic salt mixture was freshly prepared prior to the synthesis by grinding and mixing sodium chloride and zinc chloride (SZ $42 \mathrm{~mol} \%$ sodium chloride), i.e. in the ratio required for the eutectic.

In a typical synthesis of the untemplated N-S-dCs, varying amounts of the IL Emim-scn were thoroughly mixed with Emim-dca or Bmp-dca, respectively, in mass ratios of Emimscn : Emim-dca/Bmp-dca ranging from 1 to 3 with a total amount of $1 \mathrm{~g}$.

The salt templated carbons were prepared by thoroughly mixing the IL mixtures $(1 \mathrm{~g})$ with the eutectic salt mixture (SZ, $3 \mathrm{~g}$ ) prior to the calcination process.

The resulting mixtures were placed in a ceramic crucible and heated to $800{ }^{\circ} \mathrm{C}$ with a heating rate of $2.5 \mathrm{~K} \mathrm{~min}^{-1}$ in a Nabertherm N7/H Chamber Oven. After holding this temperature for $1 \mathrm{~h}$ the samples were allowed to cool to room temperature. All steps were carried out under a constant flow of nitrogen. In order to remove the residual salt porogen, the materials were ground and washed in water for several hours and finally filtered and dried in a vacuum.

The resulting template-free carbons are named as N-S-dC_IL_ $x \_T$ where IL is the respective dea-containing ionic liquid, $x$ is the mass ratio of Emim-sen to IL and $T$ is the synthesis temperature. The salt templated carbon is denoted as
N-S-dC_IL_ $x \_X Z \_r \_T$ where $\mathrm{XZ}$ is the eutectic made from zinc chloride $(\mathrm{Z})$ with $\mathrm{X}$ being sodium chloride (S) and $r$ represents the mass ratio eutectic/IL in the precursor mixture.

\section{Characterization}

WAXS-patterns were measured on a Bruker D8 Advance instrument using $\mathrm{Cu}-\mathrm{K} \alpha$-radiation. Nitrogen sorption measurements were accomplished with $\mathrm{N}_{2}$ at $77 \mathrm{~K}$ after degassing the samples at $150{ }^{\circ} \mathrm{C}$ under vacuum for 20 hours using a Quantachrome Quadrasorb SI porosimeter. The apparent surface area was calculated by applying the BrunauerEmmett-Teller (BET) model to the isotherm data points of the adsorption branch in the relative pressure range $p / p_{0}<0.11$ for the microporous materials and $p / p_{0}<0.3$ for the mesoporous materials. The pore size distribution was calculated from $\mathrm{N}_{2}$ sorption data using the nonlocal density functional theory (NLDFT) equilibrium model method for slit pores provided by Quantachrome data reduction software QuadraWin Version 5.05. TEM images were obtained using a Zeiss EM $912 \Omega$ instrument. Elemental analysis was accomplished as combustion analysis using a Vario Micro device. SEM images were obtained on a LEO 1550-Gemini instrument after sputtering with platinum. TGA was conducted with a Perkin-Elmer STA 6000 in air at a heating rate of $10 \mathrm{~K} \mathrm{~min}^{-1}$. EDX spectra were taken on the SEM with an EDX spectrometer. XPSmeasurements were made with a Kratos Axis165 system using $\mathrm{Al}-\mathrm{K} \alpha$-radiation.

\section{Results}

The materials are produced via a one-step procedure comprising the carbonization of precursor mixtures of Emimscn (Scheme 1) with Emim-dca and Bmp-dca, respectively, under a nitrogen stream (see the ESI $\dagger$ for experimental details and exact compound structures).

The IL Emim-scn was chosen as the sulfur source since the cation had already been successfully used in the synthesis of purely nitrogen-doped carbons. ${ }^{26}$ Furthermore, the thiocyanate anion is readily available by counterion exchange with NaSCN from the halide intermediates, making this IL an easily available choice. In addition, the isothiocyanate is able to undergo cycloaddition reactions with other $\mathrm{sp}^{2}$-bonded species and can thus be straightly incorporated into the carbon network. Emimscn was used without further purification. The products are

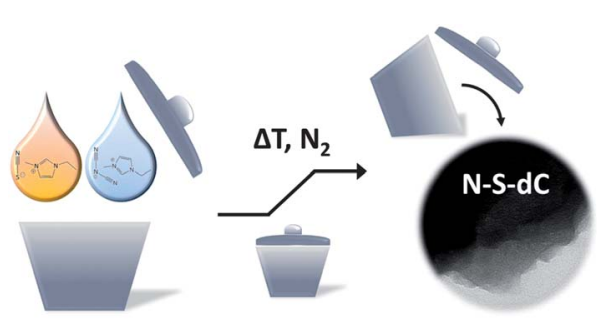

Scheme 1 Preparation of $\mathrm{N}-\mathrm{S}$-co-doped carbon through carbonization of IL mixtures containing Emim-scn as sulfur and nitrogen and Emim-dca and Bmp-dca as the nitrogen source, respectively. 
denoted as $\mathrm{N}-\mathrm{S}-\mathrm{dC} \_\mathrm{IL} \_x$ where IL is the dca-containing IL and $x$ is the mass ration of Emim-scn to IL. As can be seen from thermogravimetric analysis-differential scanning calorimetry (TGA-DSC) measurements (Fig. S- $2 \dagger$ ), carbonization of pure Emim-scn results in only low yields (Table S-1†). This was however to be expected, as the thiocyanate trimerizes into thiocyanuric acid, which does not form extended polymer networks, but is sublimated instead. Yet, Emim-scn possesses a higher temperature stability comparable to the dicyanamidecontaining ILs. In combination with Emim-dca and Bmp-dca, hetero-cycloaddition therefore sets in, and the formation of a carbonaceous product is increased (Table $\mathrm{S}-1 \dagger$ ). We attribute this to the higher polarizability of the intermediary reaction products with sulfur which results in the tunable sulfur incorporation during the onset of extended aromatization.

The powder X-ray diffraction patterns (XRD) reveal the $2 \theta \sim$ $26^{\circ}(002)$ peaks of slightly disordered graphitic carbon and the in-plane $43^{\circ}(100)$ peak, speaking for extended $\mathrm{sp}^{2}$-conjugated systems (Fig. S-3†). The in-plane peak nicely illustrates the high degree of graphitization. The position of the (002) peak, however, remains unchanged with increasing sulfur content. On the one hand, this may be attributed to sulfur mainly located at edge-terminations. On the other hand, nitrogen doping also compresses the layers (due to higher polarization interactions), thus, effects might compensate.

Concerning the bulk elemental analysis (EA), indeed a sulfur-content varying from 1.5-3 wt\% was obtained for both host ILs (Fig. 1). This amount is in the very typical range also known from other sulfur-containing carbons. ${ }^{19,26}$

The homogeneous distribution of both nitrogen and sulfur atoms throughout the sample is also shown by energy dispersive X-ray (EDX) spectroscopy (Fig. S-4†) which reveals a lower content of both heteroatom species. This is, besides some sodium residue from an incomplete ion exchange, presumably due to surface oxidation. For a decisive confirmation of the nitrogen and sulfur binding states, X-ray photoelectron spectroscopy (XPS) was performed representatively for N-S-dC_Emim-dca_2_800 and N-S-dC_Bmp-dca_2_800 (Fig. 2).

The deconvoluted $\mathrm{N}$ 1s spectra (Fig. 2, left) reveal the nitrogen atoms to be incorporated as pyridinic $(398 \mathrm{eV})$, pyrrolic $(400 \mathrm{eV})$, quaternary graphitic nitrogen $(401 \mathrm{eV})$ and oxidized nitrogen species (402 eV and $405 \mathrm{eV}$ ). This is in accordance with the results previously obtained for the purely nitrogen-doped carbons derived from the respective ionic liquid. ${ }^{21,22}$ In the detailed spectra of the $S 2 p$ orbitals, three peaks are obtained (Fig. 2, right). ${ }^{26}$ The first signal can be ascribed to sulfur atoms bound within nitrogen-aromatic rings $(163.7 \mathrm{eV})$ replacing otherwise nitrogen positions. Furthermore, contributions from aromatic carbon-sulfur motifs in the form of thiophenic sulfur species or $\mathrm{C}=\mathrm{S}$ double bonds are found (164.5-165 eV). As in the case of nitrogen, also a small amount of oxidized sulfur is observed (167 eV, $168.5 \mathrm{eV}$ ). Essentially, the XPS results reveal the nitrogen as well as sulfur atoms to be structurally integrated into the extended carbon network, thus justifying their description as nitrogen-sulfur-co-doped carbons. Furthermore, elemental analysis from XPS confirms the results obtained from the combustion method and the addition of the sulfur-containing component Emim-scn does not have any influence on the principal character of the nitrogen binding sites. Here, emphasis is put on the fact that the isothiocyanate operation mode is independent of the nature of the ionic liquid cation, thus rendering the approach a rather general method for the structural doping of carbon nanostructures with smaller amounts of sulfur.
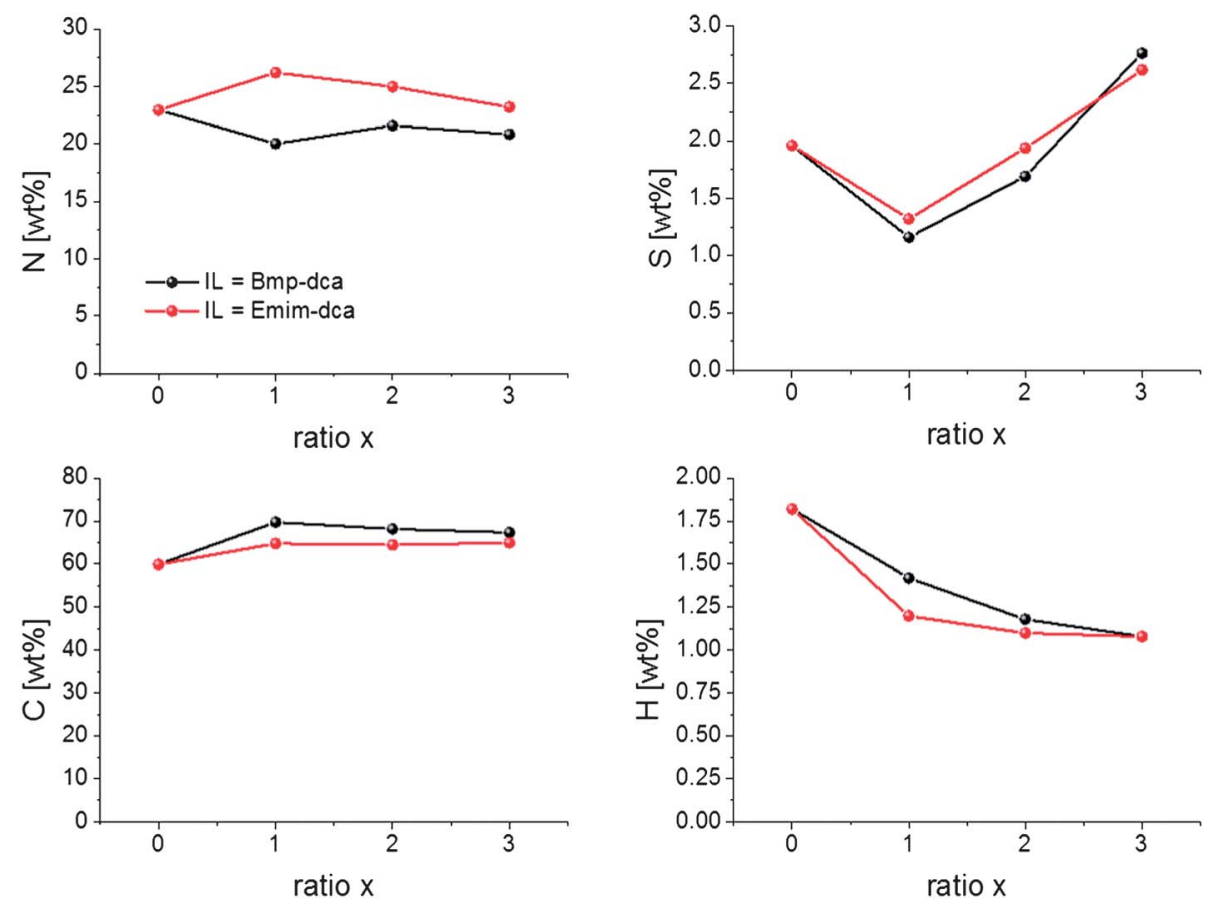

Fig. 1 Courses of N, S, C and H contents obtained from combustion EA for N-S-dC_IL_x_800 with IL = Emim-dca (red) and Bmp-dca (black). 

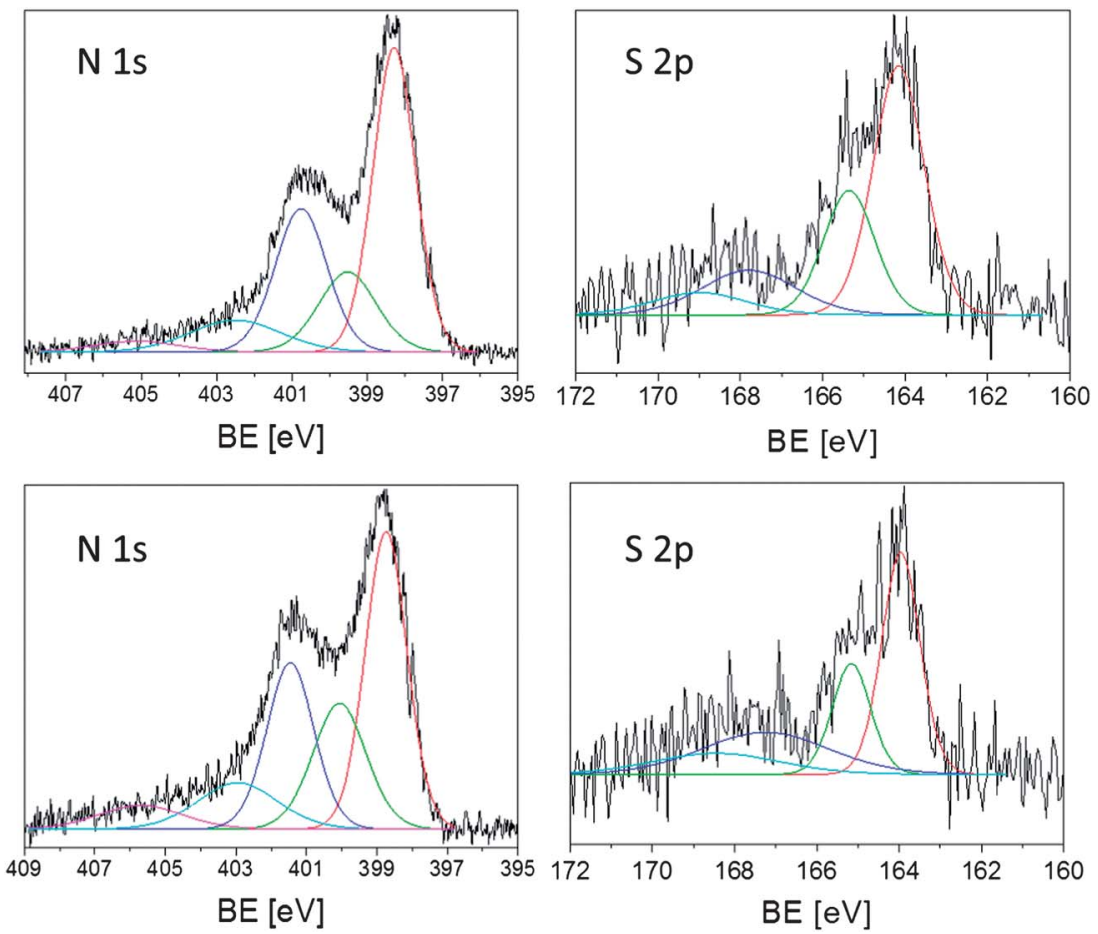

Fig. 2 XPS-spectra of the N 1s and S 2p orbitals of N-S-dC_IL_2_800 with IL = Emim-dca (top) and Bmp-dca (bottom)

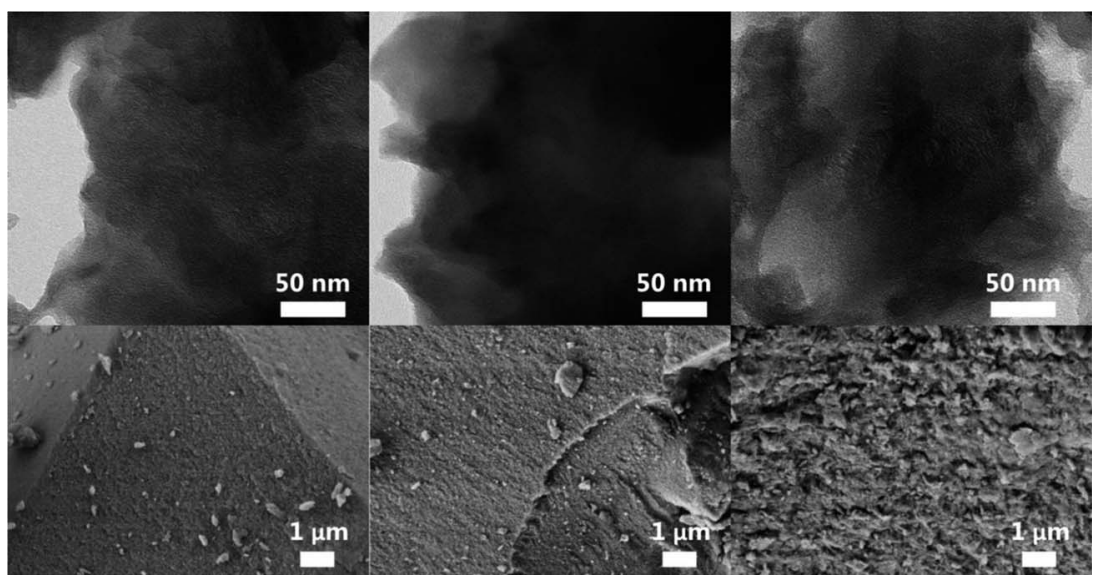

Fig. 3 TEM (top) and SEM (bottom) of $\mathrm{N}-\mathrm{S}$-dC_Emim-dca_ $x_{-} 800$ with $x=1$ (left), 2 (middle) and 3 (right).

Transmission electron micrographs (TEM, Fig. 3, top) reveal the materials to possess a bent layer structure, typically known for IL-derived nitrogen-doped carbons. This, together with observation from scanning electron microscopy (SEM, Fig. 3, bottom), nicely supports the results obtained from XRD measurements (Fig. S-3 $\dagger$ ) and accounts for the (002) peaks at $2 \theta$ $\sim 26^{\circ}$. Additionally, SEM pictures of N-S-co-doped carbons synthesized with varying amounts of Emim-scn (Fig. 3, S-5, and S-6†) show an increasing surface roughness with increasing Emim-scn content which may be ascribed to the increased sulfur content.

However, nitrogen sorption measurements reveal the $\mathrm{N}-\mathrm{S}$-co-doped carbons to possess no significant external surface area (Fig. S-7†). It has already been stated that another advantage of the ionic liquid monomer based route is the possibility to use simple salts with varying ion radii and polarizabilities as molecular templates, i.e. the salt templating approach, in order to generate carbon materials with the highest specific surface areas. ${ }^{29}$ In the present case, this would allow a rather complete access to all the heteroatom functionalities in this carbon material. Here, we exemplarily used the eutectic salt melt $\mathrm{NaCl} /$ $\mathrm{ZnCl}_{2}$ (SZ) as a template in a mass ratio of salt : IL $3: 1$ which was previously identified as a convenient protocol to synthesize high surface area carbons in high yields. XRD patterns of the products after thermal treatment show the initial formation of zinc sulfide which, however, can easily be removed by washing 

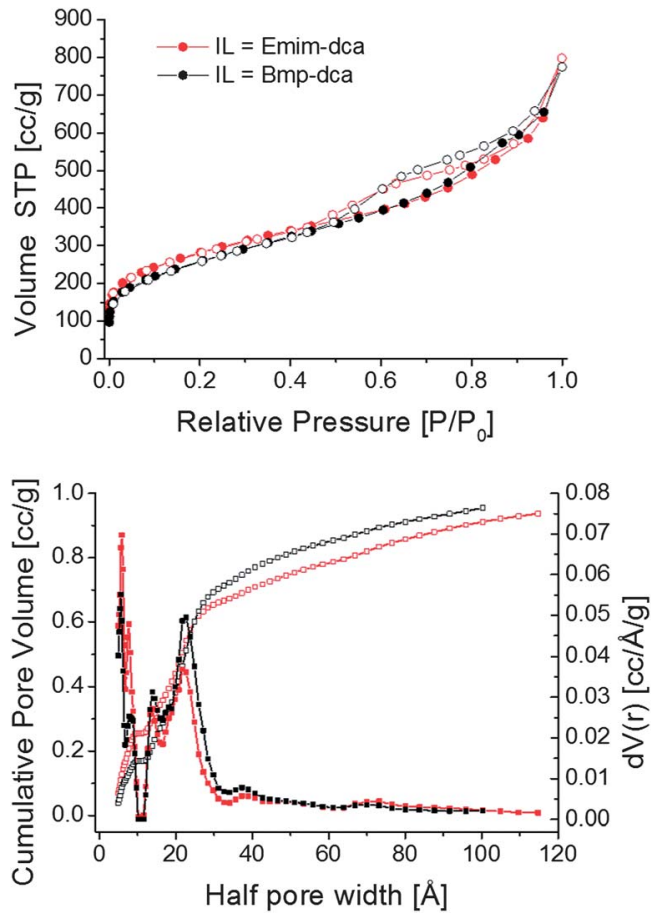

Fig. 4 Nitrogen sorption isotherms (top) and pore size distributions (bottom) of $\mathrm{N}-\mathrm{S}-\mathrm{dC}$-IL_2_SZ_3_800 with IL = Emim-dca (red) and Bmp-dca (black).

with diluted $\mathrm{HCl}$ (Fig. S-8 $\dagger$ ). SEM pictures of the washed products reveal a globular structure (Fig. S-9†). The resulting Brunauer-Emmett-Teller (BET) isotherms and pore size distributions calculated using the nonlocal density functional theory (NLDFT) are shown in Fig. 4.

The isotherms reveal high nitrogen uptake in both cases which indicates the successful generation of high porosities through the salt templating approach. Indeed, the BET surface areas are $980 \mathrm{~m}^{2} \mathrm{~g}^{-1}$ and $900 \mathrm{~m}^{2} \mathrm{~g}^{-1}$ for N-S-dC_Emimdca $\_$2_SZ_3_800 and N-S-dC_Bmp-dca_2_SZ_3_800, respectively. Furthermore, for both materials the pores sizes are 3 to $4 \mathrm{~nm}$ in diameter which is in accordance with the results previously obtained for purely nitrogen-doped and nitrogenboron-co-doped carbons. ${ }^{29}$ This also confirms the dependence of the porosity mainly on the nature of the used salt and less on the nature of the ionic liquid. It is highly relevant that compared to the respective non-porous $\mathrm{N}-\mathrm{S}$-doped carbons, the sulfur content of the salt templated carbons increased by a factor of $3(6 \mathrm{wt} \%)$, with the consequence that only half of the nitrogen (14 wt\%) is built in (Table S- $2 \uparrow$ ). This is the first time that the composition of such carbons is related to their specific surface area, that is sulfur presumably prefers thermodynamically more stable surface termination sites, while it is thermodynamically unfavorable to be incorporated within the bulk of a carbon. This interpretation goes well with the structural assignments of the XPS results and might point the way to high sulfur doped carbons. Another explanation may be given by the more efficient trapping of the thiocyanate component during the heating process due to increased electrostatic interactions with the template salt which is in accordance with the overall increased yield (Table S-1†). In any case, this illustrates another advantage of the salt templating approach where high surface areas can be introduced while the high heteroatom content can even be promoted.

We note that in a more general synthetic concept the thiocyanate could also be a part of the salt template which would result in a negatively charged, element-doped carbon or an acidic site at the carbon scaffold after water washing. In fact, this simply illustrates the general potential of the applied process to perform chemistry with carbon, presenting ILs and salt templates as parts of a combinatory toolbox.

\section{Conclusions}

In conclusion, a facile one-pot route towards nitrogen-sulfurco-doped carbons is presented. Through carbonization of ionic liquid mixtures containing Emim-sen in combination with Emim-dca and Bmp-dca, the sulfur content is easily tunable via the initial amount of the former compound. We also applied our recently reported salt templating approach to introduce high surface areas around $1000 \mathrm{~m}^{2} \mathrm{~g}^{-1}$ by simple washing with water while keeping the complete heteroatom functionality. This is rather different from standard activation processes which usually deplete the material functionality in a first step. Eventually, this opens up the way for the synthesis of carbons containing a variety of different structural heteroatoms with tunable composition. Thus, the materials are potentially suitable for several applications such as catalysts for ORR and electrodes for supercapacitors, which are subject to ongoing work.

\section{Acknowledgements}

The authors gratefully acknowledge A. Holländer and T. Greco for XPS, J. Roeser for TGA-DSC and J. Hartmann for EDX measurements.

\section{References}

1 J. Kouvetakis, R. B. Kaner, M. L. Sattler and N. Bartlett, J. Chem. Soc., Chem. Commun., 1986, 1758.

2 H. J. Burch, J. A. Davies, E. Brown, L. Hao, S. A. Contera, N. Grobert and J. F. Ryan, Appl. Phys. Lett., 2006, 89, 143110.

3 S. Glenis, A. J. Nelson and M. M. Labes, J. Appl. Phys., 1996, 80, 5404.

4 Y. Y. Shao, J. H. Sui, G. P. Yin and Y. Z. Gao, Appl. Catal., B, 2008, 79, 89.

5 Q. H. Yang, W. H. Xu, A. Tomita and T. Kyotani, Chem. Mater., 2005, 17, 2940.

6 D. P. Kim, C. L. Lin, T. Mihalisin, P. Heiney and M. M. Labes, Chem. Mater., 1991, 3, 686.

7 R. Czerw, M. Terrones, J. C. Charlier, X. Blase, B. Foley, R. Kamalakaran, N. Grobert, H. Terrones, D. Tekleab, P. M. Ajayan, W. Blau, M. Ruhle and D. L. Carroll, Nano Lett., 2001, 1, 457.

8 A. C. M. Carvalho and M. C. dos Santos, J. Appl. Phys., 2006, 100, 084305. 
9 X. Wang, J. S. Lee, Q. Zhu, J. Liu, Y. Wang and S. Dai, Chem. Mater., 2010, 22, 2178.

10 W. Yang, T.-P. Fellinger and M. Antonietti, J. Am. Chem. Soc., 2011, 133, 206.

11 T.-P. Fellinger, F. Hasché, P. Strasser and M. Antonietti, J. Am. Chem. Soc., 2012, 134, 4072.

12 L. Zhao, Z. Bacsik, N. Hedin, W. Wei, Y. Sun, M. Antonietti and M.-M. Titirici, ChemSusChem, 2010, 3, 840.

13 S. Yang, X. Feng, X. Wang and K. Müllen, Angew. Chem., Int. Ed., 2011, 50, 5339.

14 T.-P. Fellinger, D. S. Su, M. Engenhorst, D. Gautman, R. Schlögl and M. Antonietti, J. Mater. Chem., 2012, 22, 23996.

15 W. Lei, D. Portehault, R. Dimova and M. Antonietti, J. Am. Chem. Soc., 2011, 133, 7121.

16 P. F. Fulvio, J. S. Lee, R. T. Mayes, X. Wang, S. M. Mahurin and S. Dai, Phys. Chem. Chem. Phys., 2011, 13, 13486.

17 C. H. Choi, S. H. Park and S. I. Woo, J. Mater. Chem., 2012, 22, 12107.

18 G. Hasegawa, M. Aoki, K. Kanamori, K. Nakanishi, T. Hanada and K. Tadanaga, J. Mater. Chem., 2011, 21, 2060 .
19 S.-A. Wohlgemuth, F. Vilela, M.-M. Titirici and M. Antonietti, Green Chem., 2012, 14, 741.

20 J. Liang, Y. Jiao, M. Jaroniec and S. Z. Qiao, Angew. Chem., 2012, 124, 11664.

21 J. P. Paraknowitsch, A. Thomas and M. Antonietti, J. Mater. Chem., 2010, 20, 6746.

22 J. P. Paraknowitsch, J. Zhang, D. Su, A. Thomas and M. Antonietti, Adv. Mater., 2010, 22, 87.

23 J. S. Lee, H. Luo, G. A. Baker and S. Dai, Chem. Mater., 2009, 21, 4756.

24 X. Wang and S. Dai, Angew. Chem., Int. Ed., 2010, 49, 6664.

25 J. P. Paraknowitsch, Y. Zhang, B. Wienert and A. Thomas, Chem. Commun., 2013, 49, 1208.

26 J. P. Paraknowitsch, B. Wienert, Y. Zhang and A. Thomas, Chem.-Eur. J., 2012, 18, 15416.

27 S. A. Forsyth, J. M. Pringle and D. R. MacFarlane, Aust. J. Chem., 2004, 57, 113.

28 C. L. Hussey, Adv. Molten Salt Chem., 1983, 5, 185.

29 N. Fechler, T.-P. Fellinger and M. Antonietti, Adv. Mater., 2013, 25, 75 .

30 J. S. Lee, X. Wang, H. Luo, G. A. Baker and S. Dai, J. Am. Chem. Soc., 2009, 131, 4596. 